\title{
The Minimum Spanner Problem on Butterfly Graphs
}

\author{
Shien-Ching Hwang and Gen-Huey Chen \\ Department of Computer Science and Information Engineering, \\ National Taiwan University, Taipei, TAIWAN \\ Email: ghchen@csie.ntu.edu.tw
}

\begin{abstract}
Given a connected graph $G$, a spanning subgraph $G^{\prime}$ of $G$ is called a t-spanner if every pair of two adjacent vertices in $G$ has a distance of at most $t$ in $G^{\prime}$. A t-spanner of a graph $G$ is minimum if it contains minimum number of edges among all t-spanners of $G$. Finding minimum spanners for general graphs is rather difficult. Most of previous results were obtained for some particular graphs, e.g., butterfly graphs, cube-connected cycles, de Bruijn graphs, Kautz graphs, complete bipartite graphs, and permutation graphs. The butterfly graphs were originally introduced as the underlying graphs of FFT networks which can perform the fast Fourier transform (FFT) very efficiently. In this paper, we successfully construct most of the minimum $t$-spanners for the $k$-ary $r$-dimensional butterfly graphs for $2 \leq t \leq 6$ and $t=8$.
\end{abstract}

\section{Introduction}

The notion of spanners was originated from constructing synchronizers that are used to transform synchronous algorithms into asynchronous ones [6]. Given a connected graph $G$, a spanning subgraph $G^{\prime}$ of $G$ is called a $t$-spanner if $d(u, v) \leq t$ in $G^{\prime}$ for every edge $(u, v)$ of $G$, where $d(u, v)$ is the distance between $u$ and $v$. Further, a $t$-spanner $G^{\prime}$ is proper if it is a proper subgraph of $G$, and minimum if it contains minimum edges among all $t$-spanners of $G$. A $t$-spanner is a tree $t$-spanner if it forms a tree. A tree $t$-spanner must be a minimum $t$-spanner. A $t$-spanner is also a $t^{\prime}$-spanner for $t^{\prime}>t$. $G$ is a $t$-spanner of itself for $t \geq$ 1.

Finding minimum spanners, in general, is very difficult. It was shown in [7] that finding a minimum $t$-spanner of an arbitrary graph for $t \geq 2$ is NP-hard. It remains NP-hard even if the graph is of bounded degree (see [1]). Thus far, minimum $t$-spanners have been obtained for some particular graphs, e.g., butterfly graphs $(2 \leq t \leq 7)[2,3]$, cube-connected cycles $(2 \leq t \leq 7)$ [3], de Bruijn graphs $(t=$ $2,3)[2,3]$, Kautz graphs $(t=2,3)$ [2], complete bipartite graphs $(t=3)$ [5], and permutation graphs $(t=3)$ [5].

For convenience we use $B F(k, r)$ to denote an $r$-dimensional $k$-ary butterfly graph. A formal definition of $B F(k, r)$ is given in the next section. Heydemann et al. [3] first constructed a minimum 2-spanner of $B F(2,3)$ that contains 40 edges, a minimum 3-spanner of $B F(2, r)$ that contains $3 r 2^{r-1}$ edges for $r \geq 5$, a minimum $t$-spanner of $B F(2, r)$ that contains $(3 r-1) 2^{r-1}$ edges for $3 \leq r \leq 7$ and $\max \{3, r-1\} \leq t \leq 6$, and a minimum 7-spanner of $B F(2, r)$ that contains $5 r 2^{r-2}$ edges for $r \geq 9$. However, their results are not correct for $4 \leq t \leq 6$ and $3 \leq r \leq t-1$. Then, Harbane and Padró [2] constructed minimum 3-spanners of $B F(k, r)$ that contains $(2 r-1) k^{r-1}$ edges for $r \geq 5$. In this paper, we construct a minimum $t$-spanner of $B F(2, r)$ that contains $((2 r-1) k-r) k^{r-1}$ edges for $4 \leq t \leq 6$ and $r=t-2$ or $t-1$, a minimum $t$-spanner of $B F(k, r)$ that contains $(2 k$ $-1) r k^{r-1}$ edges for $3 \leq t \leq 6$ and $(k=2$ and $r \geq t+2$ or $k \geq 3$ and $r \geq t$, and a minimum 8-spanner of $B F(k, r)$ that contains $5 r 2^{r-2}$ edges for $r \geq 10$ even. Moreover, an $(r+$ 1)-spanner of $B F(k, r)$ that contains $((2 r-1) k-r) k^{r-1}$ edges is constructed as well. We show in Table $I$ the current status of the minimum $t$-spanners for $B F(k, r)$.

\section{Preliminaries}

There are $r$ levels, numbered $0,1, \ldots, r-1$, associated with $B F(k, r)$. Each level contains $k^{r}$ vertices that are labeled with $k k$-ary sequences of $r$ symbols. A vertex of level $l$ labeled with $\beta_{0} \beta_{1} \cdots \beta_{r-1}$ is represented with $<l$, $\beta_{0} \beta_{1} \cdots \beta_{r-1}>$, where $0 \leq l \leq r-1$ and $0 \leq \beta_{j} \leq k-1$ for all $0 \leq$ $j \leq r-1$. Each vertex $<l, \beta_{0} \beta_{1} \cdots \beta_{r-1}>$ is connected to $2 k$ vertices: $<l-1, \beta_{0} \beta_{1} \cdots \beta_{l-2} i \beta_{l} \cdots \beta_{r-1}>$ and $<l+1, \beta_{0} \beta_{1} \cdots \beta_{l-1} i$ $\beta_{l+1} \cdots \beta_{r-1}>$ for all $0 \leq i \leq k-1$. Throughout this paper, all arithmetic computations whose operands are the symbols and the levels are performed modulo $k$ and modulo $r$, respectively. A formal definition of $B F(k, r)$ is as follows.

Definition 1. The vertex set of $B F(k, r)$ is $\left\{<l, \beta_{0} \beta_{1} \cdots \beta_{r-1}>\right.$ $0 \leq l \leq r-1$ and $0 \leq \beta_{j} \leq k-1$ for all $\left.0 \leq j \leq r-1\right\}$. The edge set of $B F(k, r)$ is $\left\{\left(<l, \beta_{0} \beta_{1} \cdots \beta_{r_{1}}>,<l+1, \beta_{0}^{\prime} \beta_{1}^{\prime} \cdots\right.\right.$ $\left.\beta_{r-1}^{\prime}>\right) \mid 0 \leq l \leq r-1$ and $\beta_{j}=\beta_{j}^{\prime}$ for all $0 \leq j \leq r-1$ and $j \neq$ $l\} .\left(<l, \beta_{0} \beta_{1} \cdots \beta_{r-1}>,<l+1, \beta_{0}^{\prime} \beta_{1}^{\prime} \cdots \beta_{r-1}^{\prime}>\right)$ is referred to as an $i$-edge if $\beta_{l}^{\prime}=\beta_{l}+i$, where $0 \leq i \leq k-1$. An $i$-edge is further called a straight edge if $i=0$, and a skew edge if $i \neq$ 0 .

$B F(k, r)$ is regular of degree $2 k$. There are $r k^{r}$ vertices 
and $r k^{r+1}$ edges contained in $B F(k, r)$. The edges exist between adjacent levels. Level 0 and level $r-1$ are considered adjacent. Hence, $B F(k, r)$ with even $r$ is a bipartite graph. Since $B F(k, 1)$ and $B F(1, r)$ have trivial structures and $B F(k, 2)$ is a multigraph, we consider $B F(k$, $r)$ with $k \geq 2$ and $r \geq 3$ in this paper. The structure of $B F(3$, $3)$ is depicted in Figure 1, where level 0 is replicated. $B F(k$, $r$ ) thus defined was named wrapped butterfly graphs in [4].

Given $<l, \beta_{0} \beta_{1} \cdots \beta_{r-1}>$, the subgraph of $B F(k, r)$ induced by $\left\{<l, \beta_{0} \beta_{1} \cdots \beta_{l-1}\left(\beta_{l}+i\right) \beta_{l+1} \cdots \beta_{r-1}>\mid 0 \leq i \leq k-1\right\} \cup\{<l+1$, $\left.\beta_{0} \beta_{1} \cdots \beta_{l-1}\left(\beta_{l}+i\right) \beta_{l+1} \cdots \beta_{r-1}>\mid 0 \leq i \leq k-1\right\}$ is a complete bipartite graph with two partite sets of size $k$. In terms of graph notations, the subgraph is a $K_{k, k}$. In this paper we consider the subgraph a basic module of $B F(k, r)$. Three basic modules of $B F(3,3)$ are shown with bold edges in Figure 1 . The following three observations are about the basic module.

Observation 1. The edge set of $B F(k, r)$ can be partitioned into $r k^{r-1}$ basic modules.

Observation 2. Suppose $u$ is a vertex in $B F(k, r)$, and $v, w$ are two vertices of the same level that are adjacent to $u$. Then, $u, v$, and $w$ belong to the same basic module.

Observation 3. Two distinct basic modules are vertex-disjoint or have one vertex in common.

A cycle in $B F(k, r)$ is referred to as an $i$-cycle if its edges are all $i$-edges. For example, $(\langle 0,000\rangle,\langle 1,000\rangle$, $\langle 2,000\rangle,\langle 3,000\rangle,\langle 0,000\rangle)$ is a 0 -cycle in $B F(3,3)$ and $(<0,000\rangle,\langle 1,100\rangle,<2,110\rangle,<0,111\rangle,<1,211\rangle,<2$, $221\rangle,\langle 0,222\rangle,\langle 1,022\rangle,\langle 2,002\rangle,<0,000\rangle)$ is a 1 -cycle in $B F(3,3)$. Each 0 -cycle in $B F(k, r)$ contains $r$ straight edges and each 1-cycle in $B F(k, r)$ contains $k r$ skew edges. There is no skew edge in 0 -cycles, and there is no straight edge in 1-cycles.

In [5], Madanlal et al. constructed a tree 3-spanner of a $K_{k, k}$. Suppose $X=\left\{x_{1}, x_{2}, \ldots, x_{k}\right\}$ and $Y=\left\{y_{1}, y_{2}, \ldots, y_{k}\right\}$ are the two partite sets of the $K_{k, k}$. The tree 3-spanner contains edges $\left(x_{1}, y_{i}\right),\left(x_{j}, y_{j}\right)$ for $1 \leq i \leq k$ and $2 \leq j \leq k$. According to Observation 1 , a spanning subgraph of $B F(k, r)$ can result if the tree 3-spanner is constructed for each basic module. We use $S_{k, r}$ to denote the resulting spanning subgraph. Clearly, $S_{k, r}$ contains $(2 k-1) r k^{r-1}$ edges. Since a 3 -spanner is also a $t$-spanner for $t \geq 3$. We have the following lemma.

Lemma 1. $S_{k, r}$ is a $t$-spanner of $B F(k, r)$ for $t \geq 3$.

Let $T_{k, r}$ be a spanning subgraph of $B F(k, r)$ whose edge set is $\left\{\left(<0, \alpha \alpha \beta_{2} \cdots \beta_{r-1}>,<1, \beta_{0} \alpha \beta_{2} \cdots \beta_{r-1}>\right) \mid \alpha, \beta_{0}, \beta_{2}, \ldots\right.$, $\left.\beta_{r-1} \in\{0,1, \ldots, k-1\}\right\} \cup\left\{\left(<1, \alpha \alpha \beta_{2} \cdots \beta_{r-1}>,<2, \alpha \beta_{1} \beta_{2} \cdots\right.\right.$ $\left.\beta_{r-1}>\right) \mid \alpha, \beta_{1}, \beta_{2}, \ldots, \beta_{r-1} \in\{0,1, \ldots, k-1\}$ and $\left.\alpha \neq \beta_{1}\right\} \cup$ $\left\{\left(<1, \beta_{0} \beta_{1} \cdots \beta_{r-1}>,<2, \beta_{0} \beta_{1} \cdots \beta_{r-1}>\right) \mid \beta_{0}, \beta_{1}, \ldots, \beta_{r-1} \in\{0\right.$, $1, \ldots, k-1\}$ and $\left.\beta_{0} \neq \beta_{1}\right\} \cup\left\{\left(<l, \beta_{0} \beta_{1} \cdots \beta_{r-1}>,<l+1\right.\right.$, $\left.\beta_{0} \beta_{1} \cdots \beta_{r-1}>\right) \mid \beta_{0}, \beta_{1}, \ldots, \beta_{r-1} \in\{0,1, \ldots, k-1\}$ and $l \in\{2$, $3, \ldots, r-1\}\} \cup\left\{\left(<l, \beta_{0} \beta_{1} \cdots \beta_{l-1} 0 \beta_{l+1} \cdots \beta_{\mu-1}>,<l+1, \beta_{0} \beta_{1} \cdots\right.\right.$ $\left.\beta_{l-1} \beta_{l} \beta_{l+1} \cdots \beta_{r-1}>\right) \mid \beta_{0}, \beta_{1}, \ldots, \beta_{r-1} \in\{0,1, \ldots, k-1\}, l \in\{2$, $3, \ldots, r-1\}$, and $\left.\beta_{l} \neq 0\right\}$. Clearly, $T_{k, r}$ contains $((2 r-1) k-$ $r) k^{r-1}<(2 k-1) r k^{r-1}$ edges, where $(2 k-1) r k^{\mu-1}$ is the number of edges in $S_{k, r}$. The 4-spanner shown in Figure 2 is the graph $T_{2,3}$. As explained below, $T_{k, r}$ is an $(r+$ 1)-spanner of $B F(k, r)$.

The edges of $B F(k, r)$ that are not contained in $T_{k, r}$ can be classified into the following five classes: $(1)\left(<0, \beta_{0} \beta_{1} \ldots\right.$ $\left.\beta_{r-1}>,<1, \beta_{0} \beta_{1} \cdots \beta_{r-1}>\right),(2)\left(<0, \beta_{0} \beta_{1} \cdots \beta_{r-1}>,<1,\left(\beta_{0}+i\right) \beta_{1}\right.$ $\left.\beta_{2} \cdots \beta_{r-1}>\right)$, (3) $\left(<1, \beta_{0} \beta_{0} \beta_{2} \beta_{3} \cdots \beta_{r-1}>,<2, \beta_{0} \beta_{0} \beta_{2} \beta_{3} \cdots \beta_{r-1}>\right)$, (4) $\left(<1, \beta_{0} \beta_{1} \cdots \beta_{r-1}>,<2, \beta_{0}\left(\beta_{1}+i\right) \beta_{2} \cdots \beta_{r-1}>\right)$, and (5) $(<l$, $\left.\beta_{0} \beta_{1} \cdots \beta_{r-1}>,<l+1, \beta_{0} \beta_{1} \cdots \beta_{l-1}\left(\beta_{l}+i\right) \beta_{l+1} \cdots \beta_{r-1}>\right)$, where $i \epsilon$ $\{1,2, \ldots, k-1\}, \beta_{0}, \beta_{1}, \ldots, \beta_{r-1} \in\{0,1, \ldots, k-1\}, l \in\{2$, $3, \ldots, r-1\}, \beta_{0} \neq \beta_{1}$, and $\beta_{l} \neq 0$. Between both end vertices of each edge above, there is a path of length $\leq(r+1)$ in $S$. The path can be constructed as $\left(<0, \beta_{0} \beta_{1} \cdots \beta_{r-1}\right\rangle,<r-1, \beta_{0}$ $\left.\beta_{1} \cdots \beta_{r-1}>,<r-2, \beta_{0} \beta_{1} \cdots \beta_{r-1}>, \ldots,<1, \beta_{0} \beta_{1} \cdots \beta_{r-1}>\right)$ for (1), $\left(<0, \beta_{0} \beta_{1} \cdots \beta_{r-1}>,<r-1, \beta_{0} \beta_{1} \cdots \beta_{r-1}>,<r-2, \beta_{0} \beta_{1} \cdots\right.$ $\beta_{r-1}>, \ldots,<1, \beta_{0} \beta_{1} \cdots \beta_{r-1}>,<0, \beta_{1} \beta_{1} \cdots \beta_{r-1}>,<1,\left(\beta_{0}+i\right) \beta_{1} \beta_{2}$ $\left.\cdots \beta_{r-1}>\right)$ for $(2),\left(<1, \beta_{0} \beta_{0} \beta_{2} \beta_{3} \cdots \beta_{r-1}>,<0, \beta_{0} \beta_{0} \beta_{2} \beta_{3} \cdots \beta_{r-1}>\right.$, $<r-1, \beta_{0} \beta_{0} \beta_{2} \beta_{3} \cdots \beta_{r-1}>,<r-2, \beta_{0} \beta_{0} \beta_{2} \beta_{3} \cdots \beta_{r-1}>, \ldots,<2, \beta_{0}$ $\left.\beta_{0} \beta_{2} \beta_{3} \cdots \beta_{r-1}>\right)$ for (3), $\left(<1, \beta_{0} \beta_{1} \cdots \beta_{r-1}>,<2, \beta_{0} \beta_{1} \cdots \beta_{r-1}>\right.$, $<1, \beta_{0}\left(\beta_{1}+i\right) \beta_{2} \cdots \beta_{r-1}>,<0, \beta_{0}\left(\beta_{1}+i\right) \beta_{2} \cdots \beta_{r-1}>,<r-1, \beta_{0}$ $\left(\beta_{1}+i\right) \beta_{2} \cdots \beta_{r-1}>,<r-2, \beta_{0}\left(\beta_{1}+i\right) \beta_{2} \cdots \beta_{r-1}>, \ldots,<2, \beta_{0}\left(\beta_{1}\right.$ $\left.+i) \beta_{2} \cdots \beta_{r-1}>\right)$ for (4) if $\beta_{0}=\beta_{1}+i,\left(<1, \beta_{0} \beta_{1} \cdots \beta_{r-1}>,<2, \beta_{0} \beta_{1}\right.$ $\left.\cdots \beta_{r-1}>,<1, \beta_{0} \beta_{0} \beta_{2} \cdots \beta_{r-1}>,<2, \beta_{0}\left(\beta_{1}+i\right) \beta_{2} \cdots \beta_{r-1}>\right)$ for (4) if $\beta_{0} \neq \beta_{1}+i$, and $\left(<l, \beta_{0} \beta_{1} \cdots \beta_{r-1}>,<l+1, \beta_{0} \beta_{1} \cdots \beta_{r-1}>,<l, \beta_{0}\right.$ $\left.\beta_{1} \cdots \beta_{l-1} 0 \beta_{l+1} \cdots \beta_{r-1}>,<l+1, \beta_{0} \beta_{1} \cdots \beta_{l-1}\left(\beta_{l}+i\right) \beta_{l+1} \cdots \beta_{r-1}>\right)$ for (5).

\section{Minimum spanners}

Since each 0-cycle in $B F(k, r)$ has length $r$ and there is no cycle of length three in $B F(k, r)$ that contains skew edges, there is no proper 2-spanner of $B F(k, r)$ for $r \geq 4$ and there are minimum 2-spanners of $B F(k, 3)$ which can be obtained by removing a straight edge from each 0 -cycle. Each minimum 2-spanner of $B F(k, 3)$ contains $3 k^{4}-k^{3}$ edges. In the rest of this section, minimum $t$-spanners of $B F(k, r)$ for $3 \leq t \leq 6$ are constructed.

\subsection{Minimum 3-spanners}

A minimum 3-spanner of $B F(2, r)$ was constructed in [3], which contains $3 r 2^{r-1}$ edges if $r \geq 5$ and $(3 r-1) 2^{r-1}$ edges if $r=3$ or 4 . In [2], Harbane and Padró constructed 3 -spanners of the complete generalized $p$-cycle graphs, which represent a family of underlying graphs produced by the conjunction of a directed cycle of length $p$ with a de Bruijn digraph. They showed that $B F(k, r)$ belongs to the family of graphs and the 3-spanner is minimum for $B F(k, r)$ with $r \geq 5$. In the following, a minimum 3-spanner of $B F(k$, $r$ ) for $k \geq 3$ and $r \geq 3$ is constructed.

Since the skew edges in a basic module of $B F(k, r)$ together form a connected spanning subgraph of the basic module, we have the following lemma.

Lemma 2. Suppose $H$ is a disconnected spanning subgraph 
of a basic module of $B F(k, r)$, where $k \geq 3$. There is a skew edge in the basic module whose both end vertices belong to two distinct components of $H$.

Lemma 3. Every cycle of length $\leq 4$ in $B F(k, r)$ is either a 0 -cycle or contained in a basic module.

Proof. Suppose $(u, v, w, u)$ is a cycle of length three in $B F(k, r)$. Without loss of generality, we assume $\operatorname{lev}(u)=l$ and $\operatorname{lev}(v)=l+1$, where $0 \leq l \leq r-1$ and $\operatorname{lev}(u)$ represents the level of vertex $u$. Then, $\operatorname{lev}(w)=l+2$. We have $\operatorname{lev}(u)$ $=\operatorname{lev}(w)+1$ or $\operatorname{lev}(w)-1$, which implies $r=3$. Hence $(u, v$, $w, u)$ is a 0 -cycle in $B F(k, 3)$.

On the other hand, suppose $(u, v, w, x, u)$ is a cycle of length four in $B F(k, r)$. We assume $\operatorname{lev}(u)=l$ and $\operatorname{lev}(v)=l$ +1 , where $0 \leq l \leq r-1$. Then, $l e v(w)=l$ or $l+2$ and $l e v(x)$ $=l-1$ or $l+1$. If $\operatorname{lev}(w)=l$ and $\operatorname{lev}(x)=l-1$, then $u, v$, and $w$ belong to the same basic module and $w, x$, and $u$ belong to the same basic module according to Observation 2. By Observation 3 the two basic modules are identical. Hence, $(u, v, w, x, u)$ is contained in a basic module. The discussion is similar if $\operatorname{lev}(w)=l$ and $\operatorname{lev}(x)=l+1$ or $\operatorname{lev}(w)$ $=l+2$ and $\operatorname{lev}(x)=l+1$. If $\operatorname{lev}(w)=l+2$ and $\operatorname{lev}(x)=l-1$, we have $r=4$ because $w$ and $x$ are adjacent. It is easy to see that $(u, v, w, x, u)$ is a 0 -cycle in $B F(k, 4)$.

Theorem 1. A minimum 3-spanner of $B F(k, r)$, which contains $(2 k-1) r k^{r-1}$ edges, can be constructed, where $k \geq$ 3 and $r \geq 3$.

Proof. We show that $S_{k, r}$ is a minimum 3-spanner of $B F(k$, $r)$. By Lemma $1 S_{k, r}$ is a 3-spanner of $B F(k, r) . S_{k, r}$ is minimum if every 3-spanner of $B F(k, r)$ contains at least $(2 k-1) r k^{-1}$ edges.

Suppose $S$ is a 3-spanner of $B F(k, r), B$ is a basic module of $B F(k, r)$, and $B^{\prime}$ is the subgraph of $S$ induced by the vertex set of $B . B^{\prime}$ is not necessarily a 3-spanner of $B$. We first show that every skew edge $(u, v)$ in $B$ has $d(u, v)$ $\leq 3$ in $B^{\prime}$. If $(u, v)$ is an edge of $S$, then $d(u, v)=1$ in $B^{\prime}$. Otherwise, there is a path of length $\leq 3$ between $u$ and $v$ in $S$. The path together with $(u, v)$ forms a cycle of length $\leq 4$ in $B F(k, r)$. Since there is no skew edge in 0-cycles, the cycle is contained in $B$ according to Lemma 3 . Hence, the path is contained in $B^{\prime}$, i.e., $d(u, v) \leq 3$ in $B^{\prime}$.

Then we show that $B^{\prime}$ is connected. If $B^{\prime}$ is disconnected, then Lemma 2 assures a skew edge $\left(u^{\prime}, v^{\prime}\right)$ in $B$ so that $u^{\prime}$ and $v^{\prime}$ belong to two distinct components of $B^{\prime}$, which contradicts our discussion above. Hence, $B^{\prime}$ contains at least $2 k-1$ edges. By Observation $1 S$ contains at least $(2 k-1) r k^{r-1}$ edges.

\subsection{Minimum 4-spanners}

Lemma 4. There is no cycle in $B F(k, r)$ whose length is odd and smaller than $r$.

Proof. Suppose $C$ is a cycle in $B F(k, r)$ whose length is smaller than $r$. There exists a subgraph of $B F(k, r)$ whose vertices are positioned at $s$ consecutive levels so that $C$ is contained in the subgraph, where $s \leq r-1$ is even. Since the subgraph is bipartite, the length of $C$ is even.

Lemma 5. Every cycle of length five in $B F(k, 5)$ is a 0 -cycle.

Proof. Similar to the proof of Lemma 3.

Theorem 2. A minimum 4-spanner of $B F(k, r)$, which contains $(2 k-1) r k^{r-1}$ edges, can be constructed where $k \geq$ 3 and $r \geq 4$ or $k=2$ and $r \geq 6$. A minimum 4-spanner of $B F(2,3)$, which contains 28 edges, can be constructed.

Proof. We show that $S_{k, r}$ is a minimum 4-spanner of $B F(k$, $r$ ) for $k \geq 3$ and $r \geq 4$ or $k=2$ and $r \geq 6$. By Lemma $1 S_{k, r}$ is a 4-spanner of $B F(k, r) . S_{k, r}$ is minimum if every 4-spanner of $B F(k, r)$ contains at least $(2 k-1) r k^{r-1}$ edges.

Suppose $S$ is a 4-spanner of $B F(k, r), B$ is a basic module of $B F(k, r)$, and $B^{\prime}$ is the subgraph of $S$ induced by the vertex set of $B$. $B^{\prime}$ is not necessarily a 4-spanner of $B$. We first consider the situation of $k \geq 3$ and $r \geq 4$. For each skew edge $(u, v)$ in $B$, we have $d(u, v) \leq 3$ in $B^{\prime}$ for the following reason. If $(u, v)$ is not an edge of $S$, then there is a path of length $\leq 4$ between $u$ and $v$ in $S$. The path together with $(u, v)$ forms a cycle of length $\leq 5$ in $B F(k, r)$. By Lemma 4 there is no cycle of length three in $B F(k, r)$. Also there is no cycle of length five in $B F(k, r)$, as a consequence of Lemma 4, Lemma 5, and the fact that $B F(k$, 4) is bipartite. Consequently, the cycle has length four. By Lemma 3 the cycle is contained in $B$, which implies $d(u, v)$ $\leq 3$ in $B^{\prime}$. By the same argument as the proof of Theorem 1 , it can be proved that $B^{\prime}$ is connected and hence contains at least $2 k-1$ edges. By Observation $1 S$ contains at least $(2 k$ - 1) $r k^{r-1}$ edges.

Then we consider the situation of $k=2$ and $r \geq 6 . B^{\prime}$ is a 4-spanner of $B$ for the following reason. If $B^{\prime}$ is not a 4-spanner of $B$, then there is an edge $(u, v)$ in $B$ so that $d(u$, $v)>4$ in $B^{\prime}$. Since $d(u, v) \leq 4$ in $S$, there is a path of length $\leq 4$ between $u$ and $v$ in $S$. The path together with $(u, v)$ forms a cycle of length $\leq 5$ in $B F(2, r)$. The cycle is neither a 0 -cycle nor contained in $B$. By Lemma 4 the cycle has length four, which contradicts Lemma 3 . Since $B$ is isomorphic to $K_{2,2}, B^{\prime}$ contains at least 3 edges. By Observation $1 S$ contains at least $3 r 2^{r-1}$ edges.

Next we consider the situation of $k=2$ and $r=3$. B is isomorphic to $K_{2,2}$, which forms a cycle of length four, denoted by $C_{4}$. If we remove a straight edge, say $\left(<l, \beta_{0} \beta_{1}\right.$ $\beta_{2}>,<l+1, \beta_{0} \beta_{1} \beta_{2}>$ ), from a $C_{4}$ and add two straight edges $\left(<l-1, \beta_{0} \beta_{1} \beta_{2}>,<l, \beta_{0} \beta_{1} \beta_{2}>\right)$ and $\left(<l+1, \beta_{0} \beta_{1} \beta_{2}>,<l+2\right.$, $\beta_{0} \beta_{1} \beta_{2}>$ ), then a cycle of length five, denoted by $C_{5}$, results. For example, refer to Figure 2 , where $(\langle 0,000\rangle,\langle 1,100\rangle$, $<0,100\rangle,\langle 1,000\rangle,<0,000\rangle)$ is a $C_{4}$ and $(<0,100\rangle,<1$, $100\rangle,\langle 2,110\rangle,\langle 1,110\rangle,\langle 2,100\rangle,<0,100\rangle$ ) is a $C_{5}$. As explained below, the 4-spanner $T_{2,3}$ shown in Figure 2 is minimum. We only need to show that $S$ contains at least 28 edges.

Suppose $\left(u_{1}, v_{1}\right)$ and $\left(u_{2}, v_{2}\right)$ are the two skew edges in $B$. It is not difficult to see that each cycle of length $\leq 5$ in $B F(2,3)$ is a 0 -cycle or a $C_{4}$ or a $C_{5}$. A 0 -cycle contains no 
skew edges. If a $C_{4}$ (or a $C_{5}$ ) contains one of $\left(u_{1}, v_{1}\right)$ and $\left(u_{2}, v_{2}\right)$, it also contains the other. Hence, $S$ contains $\left(u_{1}, v_{1}\right)$ or $\left(u_{2}, v_{2}\right)$, for otherwise there are no paths of length $\leq 4$ between $u_{1}$ and $v_{1}$ and between $u_{2}$ and $v_{2}$ in $S$. If $S$ contains one skew edge of each basic module and two edges of each 0 -cycle, then $S$ contains 28 edges.

If there is a 0 -cycle whose one edge is contained in $S$, then there are two basic modules whose skew edges are all contained in $S$. Hence, $S$ contains at least $28-1+2=29$ edges. This can be further explained as follows. Let us consider $(<0,000\rangle,\langle 1,000\rangle,\langle 2,000\rangle,<0,000\rangle)$, which is the leftmost 0 -cycle in Figure 2. Without loss of generality, we assume that the two edges $(<0,000\rangle,<1$, $000>)$ and $(<1,000>,<2,000>)$ are not contained in $S$. Then the two skew edges $(<0,000\rangle,<1,100>)$ and $(<1$, $000>,<0,100>)$ are contained in $S$, for otherwise there are no path of length $\leq 4$ between $<0,000>$ and $<1,000\rangle$ in $S$. Hence, $(<0,000\rangle,<1,000\rangle)$ can be "offset" with one of the two skew edges. Similarly, the two skew edges $(<1$, $000>,\langle 2,010>)$ and $(<2,000\rangle,<1,010\rangle)$ are contained in $S$, and $(<1,000\rangle,<2,000\rangle)$ can be "offset" with one of them. The discussion is similar if there is a 0-cycle whose three edges are not contained in $S$.

\subsection{Minimum 5-spanners and minimum 6-spanners}

Lemma 6. Every cycle of length six in $B F(k, r)$ is either a 0 -cycle or contained in a basic module, where $r \geq 5$.

Proof. Similar to the proof of Lemma 3.

Theorem 3. A minimum 5-spanner of $B F(k, r)$, which contains $(2 k-1) r k^{\mu-1}$ edges, can be constructed, where $k \geq$ 3 and $r \geq 5$ or $k=2$ and $r \geq 7$. A minimum 5-spanner of $B F(2,3)$, which contains 28 edges, can be constructed. A minimum 5-spanner of $B F(2,4)$, which contains 80 edges, can be constructed.

Proof. By the aid of Observation 1, Lemma 1, Lemma 3, Lemma 4, Lemma 5, and Lemma 6 , it can be proved that $S_{k, r}$ is a minimum 5-spanner of $B F(k, r)$ for $k \geq 3$ and $r \geq 5$ or $k=2$ and $r \geq 7$. The proof is similar to the proof (for $k \geq$ 3 and $r \geq 4$ or $k=2$ and $r \geq 6$ ) of Theorem 2. The 4-spanner $T_{2,3}$ is a minimum 5-spanner of $B F(2,3)$, because every 5-spanner of $B F(2,3)$ contains at least 28 edges, as explained below.

Suppose $S$ is a 5 -spanner of $B F(2,3)$ and $\left(u_{1}, v_{1}\right),\left(u_{2}, v_{2}\right)$ are two skew edges of a basic module. We first show that $S$ contains at least 12 skew edges. By Observation 1 there are 12 edge-disjoint basic modules. If $S$ contains at least one skew edge of each basic module, then $S$ contains at least 12 skew edges. We only need to consider the situation that neither of $\left(u_{1}, v_{1}\right)$ and $\left(u_{2}, v_{2}\right)$ is contained in $S$. It was shown in the proof of Theorem 2 that every cycle of length $\leq 5$ in $B F(2,3)$ that contains one of $\left(u_{1}, v_{1}\right)$ and $\left(u_{2}, v_{2}\right)$ also contains the other. Hence there are a cycle of length six that contains $\left(u_{1}, v_{1}\right)$ but does not contain $\left(u_{2}, v_{2}\right)$ and a cycle of length six that contains $\left(u_{2}, v_{2}\right)$ but does not contain $\left(u_{1}, v_{1}\right)$, both in $B F(2,3)$. The two cycles are 1-cycles, and they contain two skew edges of another basic module. One of $\left(u_{1}, v_{1}\right)$ and $\left(u_{2}, v_{2}\right)$ can be "offset" with one of the two skew edges. For example, consider $\left(u_{1}, v_{1}\right)$ $=(<0,000\rangle,\langle 1,100\rangle)$ and $\left.\left.\left(u_{2}, v_{2}\right)=(<0,100\rangle,<1,000\right\rangle\right)$. Then $(<0,111\rangle,<1,011>)$ and $(<0,011\rangle,<1,111>)$, which are two skew edges of another basic module, belong to the two 1-cycles that contain $\left(u_{1}, v_{1}\right)$ and $\left(u_{2}, v_{2}\right)$, respectively. Refer to Figure 2 . One of $\left(u_{1}, v_{1}\right)$ and $\left(u_{2}, v_{2}\right)$ can be "offset" with one of $(<0,111>,<1,011>)$ and $(<0$, $011>,<1,111>)$. We say that both of $\left(u_{1}, v_{1}\right)$ and $\left(u_{2}, v_{2}\right)$ and both of $(<0,111>,<1,011>)$ and $(<0,011\rangle,<1,111>)$ are the dual of each other.

Without loss of generality, we assume that $S$ contains 12 skew edges. There are eight edge-disjoint 0 -cycles in $B F(2,3)$. If each 0 -cycle has two edges contained in $S$, then $S$ contains $12+2 \times 8=28$ edges. It was shown in the proof of Theorem 2 that if there is a 0 -cycle whose one edge is contained in $S$, then there are two basic modules whose skew edges are all contained in $S$. Since at least one of their dual skew edges is contained in $S$, one of the missing edges in the 0-cycle can be "offset" with one of the dual skew edges. For example, consider the leftmost 0 -cycle $(<0,000\rangle,\langle 1,000\rangle,<2,000\rangle,<0,000\rangle)$ in Figure 2. If the two edges $(<0,000>,<1,000>)$ and $(<1$, $000>,<2,000>$ ) are not contained in $S$, then the four skew edges $(<0,000>,<1,100>),(<0,100>,<1,000>),(<1$, $000>,<2,010>)$, and $(<1,010>,<2,000>)$ must be contained in $S$. Their dual skew edges are $(<0,011>,<1$, $111>),(<0,111>,<1,011>),(<1,101>,<2,111>)$, and $(<1,111\rangle,<2,101>)$. At least one of them is contained in $S$, for otherwise there is no path of length $\leq 5$ between $<0$, $011\rangle$ and $<1,111\rangle$. Hence one of $(<0,000\rangle,<1,000>)$ and $(<1,000\rangle,<2,000\rangle)$ can be "offset" with one of $(<0$, $011>,<1,111>),(<0,111>,<1,011>),(<1,101>,<2$, $111>)$, and $(<1,111>,<2,101>)$. The discussion is similar if there is a 0 -cycle whose three edges are not contained in $S$.

By an argument similar to the proof (for $B F(2,3)$ ) of Theorem 2 , it can be proved that the 5-spanner $T_{2,4}$, which contains 80 edges, is minimum.

Lemma 7. Every cycle of length seven in $B F(k, r)$ is a 0 -cycle, where $r \geq 6$.

Proof. Similar to the proof of Lemma 3.

Lemma 8. Suppose $S$ is a minimum $t$-spanner of a bipartite graph $G$, where $t$ is odd. Then $S$ is also a minimum $(t+$ 1)-spanner of $G$.

Proof. Suppose $S^{\prime}$ is a minimum $(t+1)$-spanner of $G$. $S^{\prime}$ is also a $t$-spanner of $G$, for otherwise there exist two adjacent vertices in $G$ whose distance is $t+1$ in $S^{\prime}$, which implies a cycle of odd length in $G$. This is a contradiction. Since $S$ is a $(t+1)$-spanner of $G$, the size of $S$ is greater than or equal to the size of $S^{\prime}$. On the other hand, since $S$ is a minimum $t$-spanner of $G$, the size of $S$ is smaller than or 
equal to the size of $S^{\prime}$. Hence, the size of $S$ is equal to the size of $S^{\prime}$.

Theorem 4. A minimum 6-spanner of $B F(k, r)$, which contains $(2 k-1) r k^{r-1}$ edges, can be constructed, where $k \geq$ 3 and $r \geq 6$ or $k=2$ and $r \geq 8$. A minimum 6-spanner of $B F(2,4)$, which contains 80 edges, can be constructed. A minimum 6-spanner of $B F(2,5)$, which contains 208 edges, can be constructed.

Proof. By the aid of Observation 1, Lemma 1, Lemma 3, Lemma 4, and Lemma 6 , it can be proved that $S_{k, r}$ is a minimum 6-spanner of $B F(k, r)$ for $k \geq 3$ and $r \geq 6$ or $k=2$ and $r \geq 8$. The proof is similar to the proof (for $k \geq 3$ and $r$ $\geq 4$ or $k=2$ and $r \geq 6$ ) of Theorem 2. Since $B F(2,4)$ is bipartite, by Theorem 3 and Lemma 8 a minimum 6-spanner of $B F(2,4)$ contains 80 edges. The 5-spanner $T_{2,4}$ is a minimum 6-spanner of $B F(2,4)$.

By an argument similar to the proof (for $B F(2,3)$ ) of Theorem 2 , it can be proved that the 6 -spanner $T_{2,5}$ which contains 208 edges, is minimum.

Theorem 1, Theorem 2, Theorem 3, and Theorem 4 can be combined as follows.

Theorem 5. A minimum $t$-spanner of $B F(k, r)$ for $3 \leq t \leq 6$, which contains $(2 k-1) r k^{r-1}$ edges, can be constructed, where $k=2$ and $r \geq t+2$ or $k \geq 3$ and $r \geq t$. A minimum $t$-spanner of $B F(2, r)$ for $4 \leq t \leq 6$, which contains ( $2 r-$ 1) $k-r) k^{r-1}$ edges, can be constructed, where $r=t-2$ or $t-$ 1.

A minimum 7-spanner of $B F(2, r)$ that contains $5 r 2^{r-2}$ edges was constructed in [3], where $r \geq 9$. Since $B F(2, r)$ with even $r$ is bipartite, a minimum 8-spanner of $B F(2, r)$ that contains $5 r 2^{r-2}$ edges can be constructed according to Lemma 8, where $r \geq 10$ is even. We show in Table I the current status of the minimum $t$-spanners for $B F(k, r)$. As shown in Table I, minimum $t$-spanners of $B F(2,3)$ for $t \leq 5$ have been obtained. A minimum 3-spanner of $B F(2,3)$ contains 32 edges. A minimum 4-spanner and a minimum 5-spanner of $B F(2,3)$ contain 28 edges. Minimum $t$-spanners of $B F(2,3)$ for $t>5$ were not obtained yet.

\section{References}

[1] L. Cai and M. Keil, "Spanners in graphs of bounded degree," Networks, vol. 24, pp. 233-249, 1994.

[2] R. Harbane and C. Padró, "Spanners of de Bruijn and Kautz graphs," Information Processing Letters, vol. 62, pp. 231-236, 1997.

[3] M. C. Heydemann, J. G. Peters, and D. Sotteau, "Spanners of hypercube-derived networks," SIAM Journal on Discrete Mathematics, vol. 9, no. 1, pp. 37-54, 1996.

[4] F. T. Leighton, Introduction to Parallel Algorithms and Architecture: Arrays.Trees.Hypercubes, Morgan Kaufman, San Mateo, CA, 1992.

[5] M. S. Madanlal, G. Venkatesan, and C. P. Rangan, "Tree 3-spanners on interval, permutation and regular bipartite graphs," Information Processing Letters, vol. 59, pp. 97-102, 1996.

[6] D. Peleg and J. D. Ullman, "An optimal synchronizer for the hypercube," Proceedings of the 20th ACM Symposium on Theory of Computing, 1987, pp. 77-85.

[7] D. Peleg and A. A. Schäffer, "Graph spanners," Journal of Graph Theory, vol. 13, no. 1, pp. 99-116, 1989.

Table I. Minimum $t$-spanners of $B F(k, r)$.

\begin{tabular}{|c|c|c|c|}
\hline$t$ & $k$ & $r$ & Number of edges \\
\hline 1 & $\geq 2$ & $\geq 3$ & $r k^{r+1}$ \\
\hline \multirow{3}{*}{2} & 2 & 3 & 40 (ref. [3]) \\
\hline & $\geq 2$ & $\geq 4$ & $r k^{r+1}$ \\
\hline & 3 & 3 & 216 \\
\hline \multirow{4}{*}{3} & \multirow{2}{*}{2} & 3,4 & $(3 r-1) 2^{r-1}$ (ref. [3]) \\
\hline & & $\geq 5$ & $3 r 2^{r-1}$ (ref. [2], [3]) \\
\hline & \multirow{2}{*}{$\geq 3$} & 3,4 & $(2 k-1) r k^{r-1}($ Theorem 1$)$ \\
\hline & & $\geq 5$ & $(2 k-1) r k^{r-1}$ (Theorem 1, ref. [2]) \\
\hline \multirow{5}{*}{4} & \multirow{3}{*}{2} & 3 & $28($ Theorem 2$)$ \\
\hline & & 4,5 & $(3 r-1) 2^{r-1}$ (ref. [3]) \\
\hline & & $\geq 6$ & $3 r 2^{r-1}$ (Theorem 2) \\
\hline & \multirow{2}{*}{$\geq 3$} & 3 & unknown \\
\hline & & $\geq 4$ & $(2 k-1) r k^{r-1}$ (Theorem 2) \\
\hline \multirow{6}{*}{5} & \multirow{4}{*}{2} & 3 & $28($ Theorem 3$)$ \\
\hline & & 4 & 80 (Theorem 3) \\
\hline & & 5,6 & $(3 r-1) 2^{r-1}$ (ref. [3]) \\
\hline & & $\geq 7$ & $3 r 2^{r-1}$ (Theorem 3$)$ \\
\hline & \multirow{2}{*}{$\geq 3$} & 3,4 & unknown \\
\hline & & $\geq 5$ & $(2 k-1) r k^{n-1}$ (Theorem 3$)$ \\
\hline \multirow{7}{*}{6} & \multirow{5}{*}{2} & 3 & unknown \\
\hline & & 4 & 80 (Theorem 4) \\
\hline & & 5 & 208 (Theorem 4) \\
\hline & & 6,7 & $(3 r-1) 2^{r-1}$ (ref. [3]) \\
\hline & & $\geq 8$ & $(2 k-1) r k^{r-1}$ (Theorem 4) \\
\hline & \multirow{2}{*}{$\geq 3$} & $3,4,5$ & unknown \\
\hline & & $\geq 6$ & $(2 k-1) r k^{r-1}$ (Theorem 4) \\
\hline \multirow{3}{*}{7} & \multirow{2}{*}{2} & $\leq 8$ & unknown \\
\hline & & $\geq 9$ & $5 r 2^{r-2}$ (ref. [3]) \\
\hline & $\geq 3$ & $\geq 3$ & unknown \\
\hline \multirow{4}{*}{8} & \multirow{3}{*}{2} & $\leq 9$ & unknown \\
\hline & & $\begin{array}{l}\geq 10 \\
\text { odd }\end{array}$ & unknown \\
\hline & & $\begin{array}{l}\geq 10 \\
\text { even }\end{array}$ & $5 r 2^{r-2}$ \\
\hline & $\geq 3$ & $\geq 3$ & unknown \\
\hline$\geq 9$ & $\geq 2$ & $\geq 3$ & unknown \\
\hline
\end{tabular}




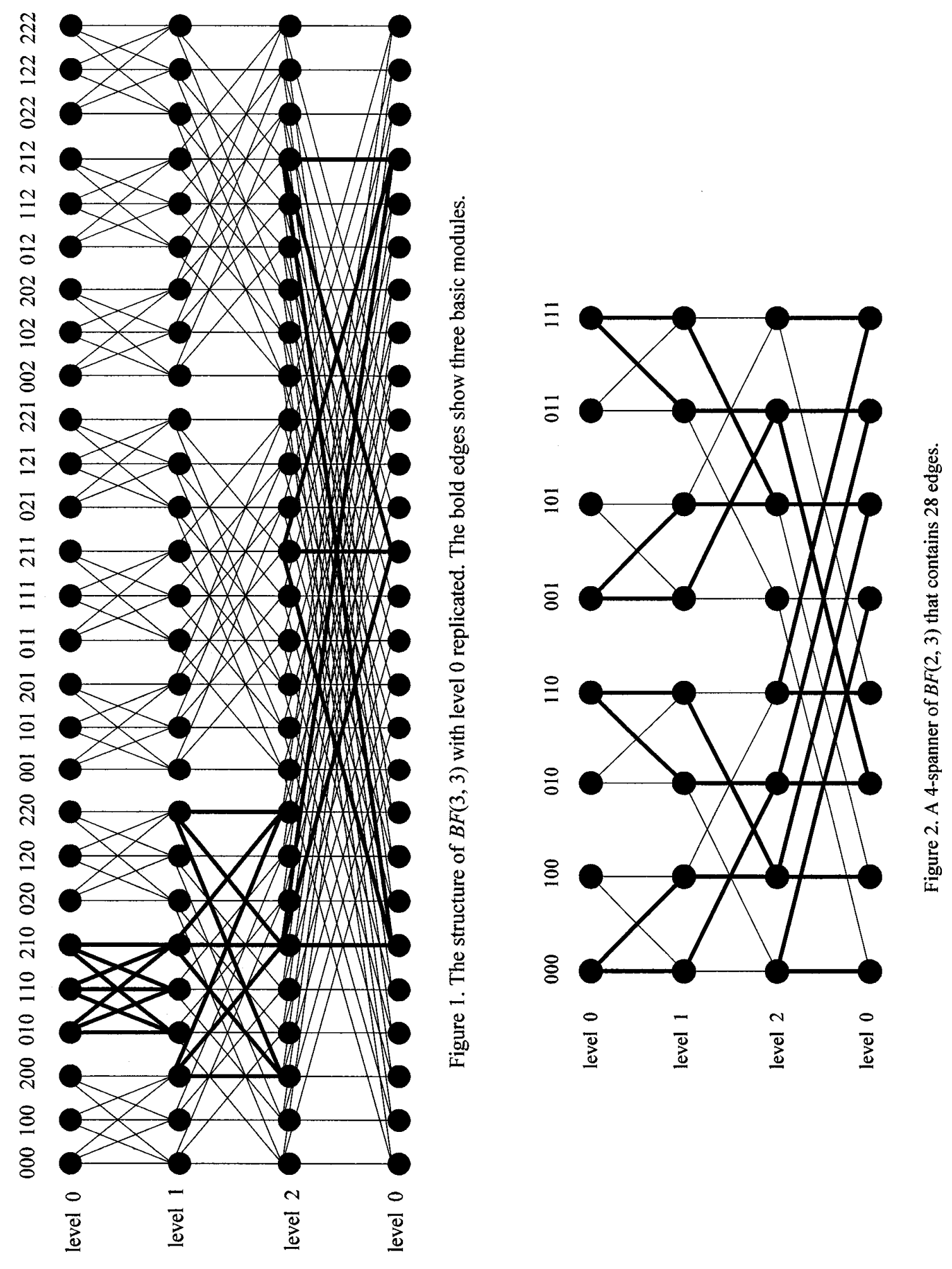

